\title{
Overview of Boron Neutron Capture Therapy: a Medical Aspect
}

\author{
Alan Anderson Bangun ${ }^{1,2, *}$ and Bagaswoto Poedjomartono ${ }^{2,3}$ \\ ${ }^{1}$ Faculty of Medicine, Diponegoro University, Semarang 50275, Indonesia \\ ${ }^{2}$ Department of Radiology, Radionuclear Services, Faculty of Medicine, Public Health, and Nursing, Universitas Gadjah Mada, \\ Yogyakarta 55281, Indonesia \\ ${ }^{3}$ Dr. Sardjito General Hospital, Jalan Kesehatan No. 1, Yogyakarta 55281, Indonesia \\ *Corresponding author: dr.alanandersonb@gmail.com
}

KEYWORDS

BNCT

Boron

Cancer

Radiation

\begin{abstract}
Cancer is an abnormal growth of a cell due to the cell's inability to control and maintain its proliferation, differentiation and apoptosis cycle. There are several methods to treat cancer; one of which is boron neutron capture therapy (BNCT). BNCT is a radiation modality by which a high radiation dose is delivered to tumor cells with lower damage to surrounding normal tissue. This modality has been used widely as a treatment for several cancer cases, such as head and neck cancer, breast cancer, and liver cancer. BNCT uses sodium borocaptate (BSH) or boronophenylalanine (BPA) as the delivery agent. Then, the tumor cell is irradiated by thermal radiation. This technique has excellent potential to become a main method of cancer therapy in the future, since it is noninvasive and has fewer side effects than other methods. Further studies on BNCT are needed to improve its performance as a cancer treatment modality.
\end{abstract}

(c) The Author(s) 2018. This article is distributed under a Creative Commons Attribution-ShareAlike 4.0 International license.

\section{INTRODUCTION}

Cancer is an abnormal growth of cells. The cell has lost its ability to control and maintain cell number normally. A normal cell divides, differentiates into another cell or does apoptosis constantly through the regular cellular signal. When cells are unable to maintain a normal life cycle, the uncontrolled cells grow and proliferate continuously. In advanced stages, cancer cells can migrate to another site in the body and invade normal tissue (Hejmadi 2010).

There are common changes in cell physiology that results in cancer. Normal cells have a finite lifespan. Cancer cells have oncogenes that trigger the cell to keep dividing by producing a protein that stimulates a proliferation of cells. Even though there is an antigrowth signal mechanism, through a tumor suppressor gene that can regulate this proliferation, cancer cells have the ability to override this mechanism resulting in avoidance of apoptosis. Cancer cells can bypass apoptosis in many ways. The most common method is by inactivating p53 protein. The mutation of p53 tumor suppressor genes results in a drastic reduction of the proapoptotic regulator. Neovascularization occurs when tumor cells release the pro-angiogenic factor. This substance will stimulate endothelial cells to activate the formation of a new blood vessel. This vessel will give the tumor cell another nutrient supply (NIH 2003).

Cancerous cells can develop from any tissue within the body. The risk factors can be divided into two large groups, internal factors, and external factors. The external factors refer to environmental factors. In the United States, twothirds of cancer cases are related to environmental exposure. Some natural substances or man-made substances may lead to DNA mutation resulting in the growth of can- cer (NIH 2003). Lifestyle plays a big role in this factor; tobacco use, excessive alcohol consumption, poor diet, inactivity, radiation, certain medical drugs, excessive exposure to sunlight (UV light), and certain viruses are environmental external factors. High environmental exposures are related to specific kind of cancer. For example, lung cancer is related to excessive exposure to asbestos and frequent tobacco use.

On the other hand, internal factors are factors inside the body that make some people have a greater tendency to develop cancer than others. Some people have an abnormality that allows them to transcript normal genes resulting in altered genes in body cells, an abnormal hormone level in the bloodstream, or a weakened immune system. This abnormality can be inherited from their ancestors. People become more susceptible to cancer due to this factor. Cancer rates are increased by the number of risks a person has. Lowering exposure to these risk factors could suppress some leading cancers. For example, the majority of lung cancer deaths are caused by tobacco use (71\%). By avoiding tobacco use, people reduce their likelihood of being threatened by lung cancer. The combination of environmental and behavioral risks (e.g. high body mass index, low fruit and vegetable intake, physical inactivity, unsafe sex etc.) - are responsible for the increasing number of cancer deaths (WHO 2009).

According to Global Burden Cancer/GLOBOCAN 2012, there were approximately 14.1 million cancer cases and 8.2 million cancer-related deaths in 2012 (Ferlay et al. 2015). There are some types of cancers that need special attention because they are commonly diagnosed worldwide. They are lung cancer (13\% of the total cases), breast cancer $(11.9 \%)$ and colorectum cancer (9.7\%) (American Can- 
cer Society 2018). Nowadays, cancer is one of the top three diseases that kills patients; it is followed by coronary heart disease and all kind of strokes. In Indonesia, there were 195,300 deaths caused by cancer in 2014 (WHO 2014). Based on Cancer Mortality Profile by (WHO 2014), trachea, bronchus, and lung cancer were the first causes of male cancer patients' mortality (Figure 1), and breast cancer was the first cause of cancer mortality in females (Figure 2).

There are several treatment modalities in treating cancer such as surgery, chemotherapy, and radiotherapy. Surgical treatment is effective in primary tumors, but it is limited to surgically sizeable and approachable tumors. Chemotherapy is the use of the chemical substance in certain dosage to fight cancer cells. The drug circulates through blood vessels to kill malignant cells. It has significant side effects due to the drug toxicity to normal cells, and it is subject to the development of resistance by the cancer cells. Radiation is a physical agent, which is used to destroy cancer cells. A high-energy ionization particle, e.g. $\mathrm{X}$-rays, gamma rays or electrons, is utilized during radiotherapy treatment. This high energy can kill cancer cells or cause genetic changes resulting in cancer cell death (Baskar et al. 2012).

Nowadays, there is another branch of radiotherapy that can be used as an alternative method to treat cancer called nuclear medicine. This therapeutic modality utilizes radiobiologic burden to harm the lesions. There are two things that always should be noticed while prescribing radiopharmaceutical dosage. They are maximal tolerable dose (MTD) of organ and maximal tolerable activity (MTA) of the individual. In oncology, the doses must be within MTDs of the limiting organ, and the administered activities must not exceed the MTA of an individual patient. It is fundamental for personalized medicine. The administration of therapeutic radiopharmaceutical substances may be given directly to the lesions or systematically to lesions, through intravenous, or orally (Siwi and Harto 2014).

One nuclear medicine modality that can be used as a cancer treatment is boron neutron capture therapy (BNCT). This therapeutic modality is a promising way to treat patients with cancer. In this method, Boron (Bo-10) is initially carried to a tumor by its oncotropic vector. Then the tumor is irradiated with thermal neutron beams producing Li-7 and He-4 ion (Nedunchezhian et al. 2016). A dense ionization is produced by $\mathrm{He}-4$ and $\alpha$ particle along its path. This radiation has the lethal energy to kill can-

Males

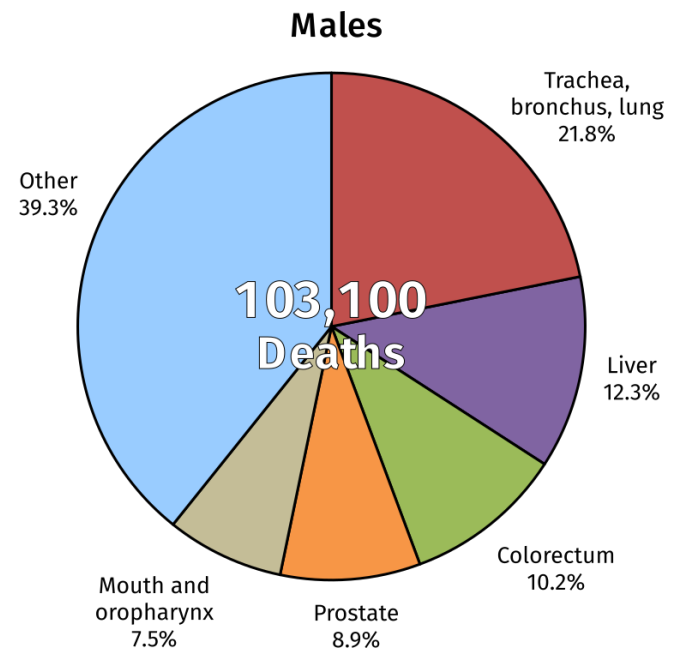

FIGURE 1. Cancer mortality profile of males in Indonesia in 2014 (WHO 2014). cer cells. sodium borocaptate (BSA), boronophenylalanine (BPA), Curcuma, and NPs loaded with targeting agents are some oncotropic vectors that can be used in this treatment (Pranoto et al. 2016).

\section{MATERIALS AND METHODS}

This study used library research method and processed primary data only. Data sources were obtained from journals, articles, books, and documentation. Relevant cases were used as a reference and processed into new information. The literature was accessed using PubMed, Elsevier, NCBI, Science Direct and Google Scholar to search for research studies published between 2000 and 2018. The keywords included cancer, radiation, BNCT, boron or a combination of these terms. This study clearly describes an overview of using BNCT in medical field based on the previous researches and studies. The objective of this study was to know the benefit of using BNCT as a cancer treatment, and furthermore to see its potency as a treatment modality.

\section{RESULTS AND DISCUSSION}

BNCT is a noninvasive method of treating malignant tumors. BNCT is based on two phenomena. First, the tumor tissue is injected with a non-radioactive boron drug. After that, the patient is irradiated with epithermal neutrons until the normal tissue dose limit is reached. When the nonradioactive boron atom captures the thermalized neutron, there will be a decaying process where the thermalized neutron decays through short-range $\mathrm{u}$-particle and recoiling Li-7 nucleus (Kageji et al. 2014). These emitted charged particles can travel only a short distance $(10 \mu \mathrm{m})$, have a high linear energy transfer, and have an associated high relative biological effectiveness (Wang et al. 2018). This energy has the ability to locally destroy the cancer cell that contains the boron drug without harming the healthy tissue around the tumor cell. To reach a good result, there are two parameters that should be considered, namely boron concentration and neutron beam.

Boron is a delivery agent that has a fundamental part in the BNCT method. Good agents are those that can be highly concentrated in a tumor cell but leave only a small residue in normal tissue. There are three main requirements to meet to be a successful delivery agent; they are (a) a high tumor uptake with a low systemic toxicity; (b) a

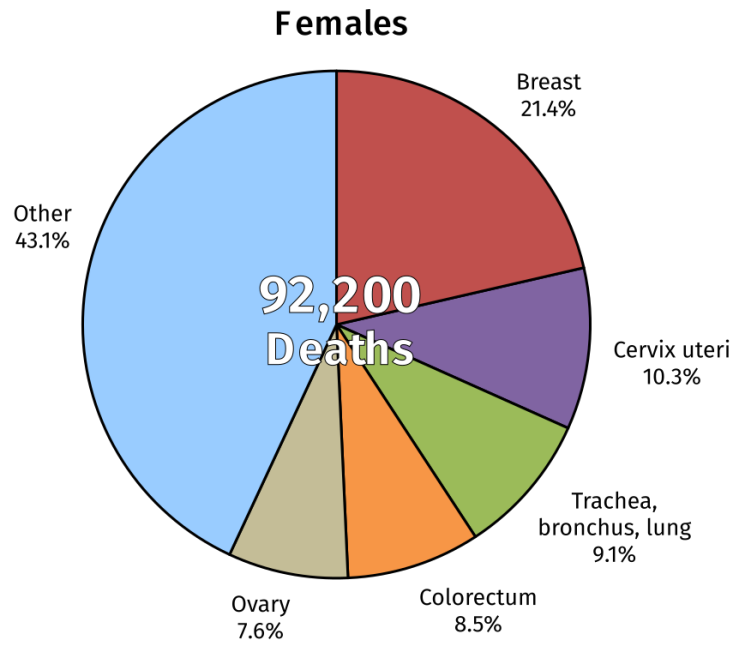

FIGURE 2. Cancer mortality profile of females in Indonesia in 2014 (WHO 2014). 
tumor concentration of $20 \mu \mathrm{g}{ }^{10} \mathrm{~B} / \mathrm{g}$ tumor; (c) and having a good persistency during BNCT but having a rapid clearance from blood vessels and normal tissues (Baskar et al. 2012). BSH (sodium boroncaptate) and BPA (boron phenylamine) are two boron compounds that are commonly used for BNCT treatment. The compounds accumulate in different places when inserted into the body. BSH will accumulate in the cell membrane while the BPA will accumulate in the nucleus of cells. BSH can pass through the disrupted blood-brain barrier easily and boron can be internalized into cells in endocytic pathways after binding to the plasma membrane. On the other side, BPA is inserted into tumor cells via the metabolism pathway of amino acids (Savolainen et al. 2013).

Boron neutron capture reaction uses thermal neutrons as they can be very effective for the treatment of tumors. Reactors are suitable for neutron source because they provide the correct energy spectrum and adequate thermal high-intensity neutron flux. Neutron source provides an epithermal spectrum ranging from $0.4 \mathrm{eV}$ to $10 \mathrm{keV}$. It is suitable for clinical use for the treatment of deep-seated tumors such as gliomas (Barth et al. 2012).

There are a lot of benefits in using BNCT as a treatment modality of cancer since BNCT is a biologically targeted type of radiation treatment. BNCT provides a better way to deliver the radiopharmaceutical dose to cure the malignant cell. Compared to the conventional radiotherapy, BNCT targets tumor cells selectively while radiotherapy uses external radiation that irradiates not only the tumor cells but also normal cells. High-energy radiation damages genetic material (deoxyribonucleic acid) of cells and thus blocks their ability to multiply and proliferate. In radiotherapy, ionizing radiation deposits energy in the cells of the tissues it passes through, which means it is not only damaging the cancer cells but also the normal cell tissues surrounding them. However, the radiotherapy is used because it is still able to control the growth of cancer cells. Effective means cancer cells receive the optimum dosage of radiation so that it can treat and control the growth adequately. Moreover, effectiveness itself cannot be separated from the safe limit (Sardjono et al. 2016). BNCT has a better effectiveness to target tumor cells than radiosurgery for treating patients with either primary or advanced stage brain tumors.

As a palliative treatment, BNCT shows a good clinical response based on the previous studies of patients who had been given treatment using BNCT alone or a combination of BNCT and other modalities (Barth et al. 2012).

In some clinical trials and investigations, an improvement was observed in cancer patients treated with BNCT. This technique can be used in newly diagnosed cancer cases as well as recurrent cases. Kankaanranta et al. (2012) reported that 22 patients with recurrent glioblastoma multiforme who had a recurrence following standard treatment were treated with BNCT using BPA. Based on the findings of study, patients with recurrent tumors can be treated using BNCT.

In the first decade of the 2000s, some clinical studies of BNCT for newly diagnosed malignant gliomas were reported. It showed a quite impressive result, where the mean survival time in each study was approximately 13 months. Combination BNCT and X-Ray therapy as cancer treatment showed a significant improvement. A better result was shown in patients with a combination of BNCT and X-Ray therapy. The mean survival time (MST) in patients with combination BNCT and X-Ray therapy was 23.5 months (Miyatake et al. 2016).
BNCT has been used widely as a treatment on severe glioma. Recently, this method has been used for treating patients with recurrent head and neck tumors who show a lack of health status improvement after receiving conventional cancer therapy.

In 2002, Miyatake et al. started their research regarding the treatment of 140 patients with brain tumors using BNCT. They had treated 32 patients with severe recurrent meningiomas, 50 patients with recurrent tumors, and 58 patients who had newly been diagnosed with advanced stage gliomas. The results are summarized as follows. BSH and L-BPA were used as a combination of delivery agents to 50 patients with severe recurrent gliomas. During the follow-up period, two-thirds of the patients showed an improvement. There was a gradual decrease in their tumor size (Figure 3). The mean survival time showed an impressive prolongation with those receiving BNCT surviving 9.1 months versus 4.4 months for the 22 patients with recurrent gliomas who received other treatment modalities (Miyatake et al. 2005).

Another report by Kawabata said that there was a reduction in tumor mass after BNCT. Nineteen patients with recurrent malignant meningioma were subjected to BNCT. There was more than $50 \%$ tumor mass reduction in 18 out of 19 cases, within the observation period. The median survival time post-BNCT was reported to be 14.1 months. BNCT provides a better option to treat malignancy successfully. This report shows that there is a great potential for using BNCT either as a first-line therapy or combined with other therapies in the future.

Patients with recurrent tumors of the head and neck region have been treated using BNCT in several countries e.g. Finland, Japan, and Taiwan. Most of them had previously had surgical treatment combined with chemo-radiation. Although the total number of patients treated by BNCT was quite small, they show some good results. Wang et al. (2018) reported there were high responses in patients with recurrent head and neck tumors (12 out of a total of 17 patients) who received BNCT treatment. Recurrence was still common especially in the area near the treatment site. This could be caused by the non-homogenous uptake of BPA-F with poor distribution in some parts of the tumors (Barth et al. 2018). To improve the clinical response of this method, further evaluation and research is needed to optimize the agent's dosage and better distribution of the agent's deliv-

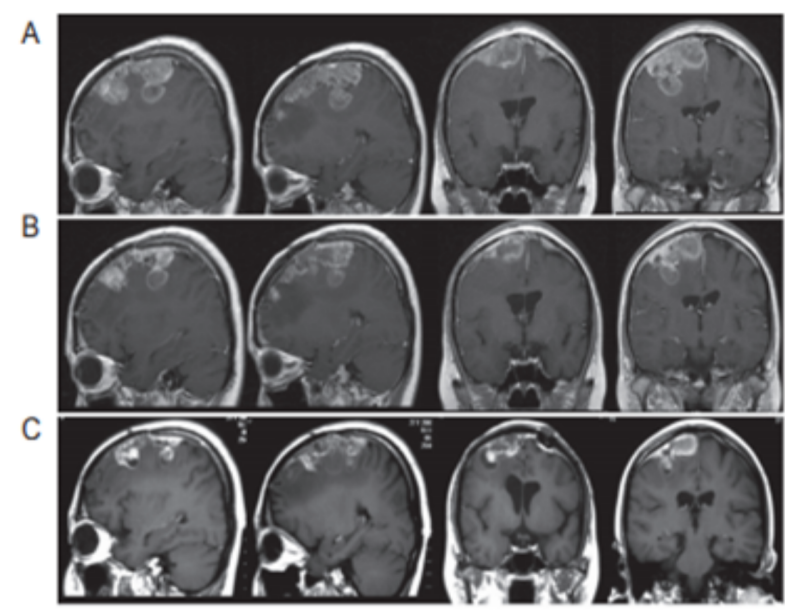

FIGURE 3. A 25-year-old woman who had recurrent rhabdoid meningioma was treated with BNCT (Miyatake et al. 2016). Her tumor size was reduced gradually in the four months following BNCT. A: one week prior to BNCT; B: two weeks after BNCT; C: four months after BNCT. 
ery. Biodistribution studies and pretreatment biopsies of other recurrent tumor sites could be very useful for improving therapeutic results.

The research on Boron Neutron Capture Therapy (BNCT) gives a great result in clinical studies, even though it has progressed slowly (Heydari and Ahmadi 2015). The development of a BNCT agent is focused on the specific target on certain cancer cells. Biomarkers become a modality to develop better agents since some cancer cells have their own specific biomarker e.g. ovarian cancer (CA-125), breast cancer (CA 15-3, CA 27-29), gastric cancer (CEA, CA 19-9, CA 72-4), prostate cancer (PSA), testicular cancer (hCG, lactate dehydrogenase), or hepatocellular carcinoma (AFP) (Bădilă et al. 2014).

Nowadays, the development of a delivery agent is limited to cancer with specific biomarkers. This biomarker may be a ligand protein or receptor in the tumor cell. Biomarkers have become an important aspect in developing the BNCT method since this method is a specific targeted therapy. Some types of cancer could be treated with BNCT because they have a specific molecular target e.g. brain tumor (epidermal growth factor receptor), breast cancer (epidermal growth factor receptor 2/HER2), Glioma (translocator protein/TSPO), etc. (Meiyanto et al. 2014). Several types of cancer do not have specific biomarkers, so it is not possible yet to use the BNCT method to treat all kinds of cancer. Further research is needed to find and develop biomarkers in many types of cancer so this method could be more applicable.

To reach an optimal clinical application for patients with cancer, the development of BNCT needs much more attention from many aspects. Further research is needed to develop and invent more selective and efficient delivery agents (boron), to calculate the dosage of agents accurately, and to improve BNCT implementation through a randomized clinical trial. The improvements will definitely allow this method to be ready to compete with other cancer treatment modalities.

\section{CONCLUSIONS}

Boron Neutron Capture Therapy is a good method of therapy to destroy malignant tumors specifically. This technique can be an alternative way to treat cancer besides surgery, chemotherapy or external radiation. There are many reports that show improvements to cancer patients after receiving treatment with BNCT. This method can be used as therapy to treat primary or recurrent cancer in many stages of severity. Even though BNCT is still new in the medical world as a form of cancer therapy, it holds promise for the future. Further evaluation and research should be done to improve therapeutic results.

\section{Acknowledgements}

The authors would like to thank everyone who contributed to this research, especially the Center of Accelerator Science and Technology, National Nuclear Energy Agency of Indonesia, who provided us with the opportunity to take part in this research.

\section{REFERENCES}

American Cancer Society. 2018. Cancer facts and figures 2018. Atlanta: American Cancer Society. https://www. cancer.org/content/dam/cancer-org/research/can cer-facts-and-statistics /annual-cancer-facts-and-f igures/2018/cancer-facts-and-figures-2018.pdf.
Barth RF, H Vicente MG, Harling OK, Kiger W, Riley KJ, Binns PJ, Wagner FM, Suzuki M, Aihara T, Kato I, Kawabata S. 2012. Current status of boron neutron capture therapy of high grade gliomas and recurrent head and neck cancer. Radiat Oncol. 7(1):146. doi:10.1186/1748-7 $17 \mathrm{X}-7-146$.

Barth RF, Zhang Z, Liu T. 2018. A realistic appraisal of boron neutron capture therapy as a cancer treatment modality. Cancer Commun. 38(1). doi:10.1186/s40880-018-0 280-5.

Baskar R, Lee KA, Yeo R, Yeoh KW. 2012. Cancer and radiation therapy: current advances and future directions. Int J Med Sci. 9(3):193-199. doi:10.7150/ijms.3635.

Bădilă E, Japie C, Bartoş D. 2014. Cancer biomarkers in clinical practice. Rom J Intern Med. 52(4):223-232.

Ferlay J, Soerjomataram I, Dikshit R, Eser S, Mathers C, Rebelo M, Parkin DM, Forman D, Bray F. 2015. Cancer incidence and mortality worldwide: Sources, methods and major patterns in GLOBOCAN 2012. Int J Cancer. 136(5):E359-E386. doi:10.1002/ijc.29210.

Hejmadi M. 2010. Introduction to Cancer Biology. 2nd edition. Copenhagen: Bookboon. https://bookboon.com /en/introduction-to-cancer-biology-ebook.

Heydari F, Ahmadi R. 2015. Tehran research reactor as a neutron source for boron neutron capture therapy in Iran: a cancer treatment plan for future. In: International Conference on Advances in Agricultural, Biological \& Environmental Sciences (AABES-2015). London: International Institute of Chemical, Biological \& Environmental Engineering. doi:10.15242/IICBE.C0715125.

Kageji T, Nagahiro S, Mizobuchi Y, Matsuzaki K, Nakagawa Y, Kumada H. 2014. Boron neutron capture therapy (BNCT) for newly-diagnosed glioblastoma: comparison of clinical results obtained with BNCT and conventional treatment. J Med Invest. 61(3-4):254-263. doi:10.2152/ jmi.61.254.

Kankaanranta L, Seppälä T, Koivunoro H, Saarilahti K, Atula T, Collan J, Salli E, Kortesniemi M, Uusi-Simola J, Välimäki P, et al. 2012. Boron neutron capture therapy in the treatment of locally recurred head-and-neck cancer: final analysis of a phase I/II trial. Int J Radiat Oncol Biol Phys. 82(1):e67-e75. doi:10.1016/j.ijrobp.201 0.09.057.

Meiyanto E, Putri H, Larasati YA, Fajarwati R. 2014. Development of boron carrying pharmaceuticals for BNCT based-cancer therapy. Book I: physics, reactors. Yogyakarta: Center for Accelerator Science and Technology, National Nuclear Energy Agency. p. 266.

Miyatake SI, Kawabata S, Hiramatsu R, Kuroiwa T, Suzuki M, Kondo N, Ono K. 2016. Boron neutron capture therapy for malignant brain tumors. Neurol Med Chir (Tokyo). 56(7):361-371. doi:10.2176/nmc.ra.2015-0297.

Miyatake SI, Kawabata S, Kajimoto Y, Aoki A, Yokoyama K, Yamada M, Kuroiwa T, Tsuji M, Imahori Y, Kirihata M, et al. 2005. Modified boron neutron capture therapy for malignant gliomas performed using epithermal neutron and two boron compounds with different accumulation mechanisms: an efficacy study based on findings on neuroimages. J Neurosurg. 103(6):1000-1009. doi:10.3171/jns.2005.103.6.1000.

Nedunchezhian K, Aswath N, Thiruppathy M, Thirugnanamurthy S. 2016. Boron neutron capture therapy - a literature review. J Clin Diagn Res. 10(12):ZE01-ZE04. doi:10.7860/JCDR/2016/19890.9024.

[NIH] National Institutes of Health. 2003. Cancer and the environment: what you need to know, what you can 
do. Technical Report NIH Publication No. 03-2039. Bethesda: National Institutes of Health. https://ww w.niehs.nih.gov/health/materials /cancer_and_the _environment_508.pdf.

Pranoto S, Maslebu G, Trihandaru S. 2016. The application of nuclear medicine. Indones J Phys Nucl Appl. 1(2):81. doi:10.24246/ijpna.vli2.81-84.

Sardjono Y, Widodo S, Irhas I, Tantawy H. 2016. A design of boron neutron capture therapy for cancer treatment in indonesia. Indones J Phys Nucl App. 1(1):1. doi:10.24246 /ijpna.v1i1.1-13.

Savolainen S, Kortesniemi M, Timonen M, Reijonen V, Kuusela L, Uusi-Simola J, Salli E, Koivunoro H, Seppälä T, Lönnroth N, et al. 2013. Boron neutron capture therapy (BNCT) in finland: technological and physical prospects after 20 years of experiences. Physica Med. 29(3):233248. doi:10.1016/j.ejmp.2012.04.008.

Siwi D, Harto A. 2014. The analysis of critical organ dose in glioblastoma therapy with boron neutron capture therapy using simulation of MNCP5. In: Y Sardjono, editor. Status boron neutron capture cancer therapy di indonesia: principles and application. Yogyakarta: Jogja Bangkit Publisher.

Wang LW, Liu YWH, Chou FI, Jiang SH. 2018. Clinical trials for treating recurrent head and neck cancer with boron neutron capture therapy using the Tsing-Hua Open Pool Reactor. Cancer Commun. 38(1). doi:10.1 186/s40880-018-0295-y.

[WHO] World Health Organization. 2009. Global health risks: mortality and burden of disease attributable to selected major risks. Geneva: World Health Organization.

[WHO] World Health Organization. 2014. WHO - Cancer country profiles Indonesia 2014. https://www.iccp-p ortal.org/who-cancer-country-profiles-indonesia2014. 